\title{
Cultura Cultura
}

Revista de Historia Teroria das ldeias $\quad$ Revista de História e Teoria das Ideias

Vol. $34 \mid 2015$

Diderot et la morale

\section{Satire et morale dans Le Neveu de Rameau}

Jean-Claude Bourdin

\section{OpenEdition}

Journals

Édition électronique

URL : http://journals.openedition.org/cultura/2465

DOI : $10.4000 /$ cultura.2465

ISSN : 2183-2021

\section{Éditeur}

Centro de História da Cultura

\section{Édition imprimée}

Date de publication : 9 juin 2015

Pagination : 135-149

ISSN : 0870-4546

Référence électronique

Jean-Claude Bourdin, « Satire et morale dans Le Neveu de Rameau », Cultura [Online], Vol. 34 | 2015 , posto online no dia 12 julho 2016, consultado a 03 maio 2019. URL : http://journals.openedition.org/ cultura/2465; DOI : 10.4000/cultura.2465

Ce document a été généré automatiquement le 3 mai 2019.

(c) CHAM - Centro de Humanidades / Centre for the Humanities 


\title{
Satire et morale dans Le Neveu de Rameau
}

\author{
Jean-Claude Bourdin
}

\section{NOTE DE L'AUTEUR}

L'édition utilisée est celle de Michel Delon, Gallimard Folio-Classique, 2006. Les références, insérées dans l'article, proviennent de cette édition dont la ponctuation est respectée. Celles concernant d'autres textes de Diderot sont données dans l'édition de Laurent Versini, Robert Laffont, « Bouquins », 1994-1997, 5 vol., abrégée en Ver, suivi du tome et de la page, également insérées dans l'article. L'Encyclopédie de Diderot et d'Alembert est citée, entre parenthèses, avec indication du tome en chiffres romains et la page en chiffres arabes. La référence sur internet est http://encyclopedie.uchicago.edu/.

1 Comme l'ensemble de l'oeuvre de Diderot Le Neveu de Rameau est entièrement domine par la morale qui attire à elle toutes les questions rencontrées et débattues entre Moi et Lui. Le dispositif formel de la conversation et le genre littéraire de la satire permettent de mélanger les sujets, les tons des répliques, les passages en prose, les didascalies et les descriptions de pantomime : il en résulte une séduisante incertitude quant à la structure du dialogue et à ce que Diderot a finalement voulu dire. ${ }^{1}$ Mais il n'y a pas de question agitée lors des échanges qui ne se ramène, très explicitement ou obliquement, à la morale. Même le long passage consacré à la musique mené de bout en bout par le neveu du grand Rameau est suivi immédiatement de la question de savoir pourquoi sa sensibilité pour les beautés de la musique et du chant s'accompagne d'une insensibilité pour les beautés de la vertu (132), de même que plus haut Moi se scandalisait de son goût et de son admiration pour les récits d'atroces forfaits $(118,119)$.

Une des façons privilégiées de comprendre les lois et les normes est de partir de l'analyse de leur dysfonctionnement, d'en venir à la norme en passant par sa transgression, au normal en passant par le pathologique ou le monstrueux ; de même connaître l'homme normal passe par la connaissance des « monstres » en tout genre ou de la prise en compte 
chez tous de la "fêlure originelle " (Ver, V, 838) qui ne cesse pas de travailler les subjectivités. Diderot est de ceux qui font bénéficier la réflexion morale de la surestimation épistémologique du singulier dans l'étude du vivant qu'il a apprise chez Buffon, pour qui les singularités et les apparentes irrégularités ont le privilège de montrer la nature " plus à découvert ${ }^{2}$ ». D'où l'intérêt insistant pour la tératologie, les fortes passions, les grands crimes, les situations étranges suscitant des cas de conscience et les individus originaux doués d'une "individualité naturelle ", comme le neveu de Rameau (47). ${ }^{3}$ Outre un goût littéraire et philosophique pour les réalités singulières qui échappent aux classifications, on a là la conséquence d'une démarche et d'une méthode : l'écart, la déviation, l'échec renseignent sur la régularité et apprennent à la relativiser. On sait les développements métaphysiques qu'il en a tirés dans son matérialisme : l'ordre actuel est un arrangement provisoire et précaire d'un désordre créateur où le multiple et le singulier sont les seules réalités qui résistent à toute analyse ou réduction. En décrivant des vicieux, la satire permet, au delà de la dénonciation morale qu'elle entraîne, d'étudier les écarts par rapport à la norme morale et invite à s'interroger sur les fondements de celle-ci, voire sur ses limites. Mais avec Diderot il faut s'attendre à ce qu'il ne fasse rien comme tout le monde : en écrivant une satire il montre en même temps que ce genre est arrivé à un terme. Les caractéristiques du monde révélé par le dialogue annulent par avance toute satire contre lui dirigée. La morale n'a plus besoin d'une satire. Il est temps de passer à ce que le siècle suivant pratiquera sous le nom de critique.

\section{La « satyre seconde » est-elle une satire?}

3 La satire est un genre qui s'attache à dénoncer tout ce qui contrevient à la moralité commune, aux valeurs et aux usages codifiés ou non. Elle a bien comme motivation de « poursuivre un vicieux » comme le dit le Paradoxe sur le comédien (Ver, IV, 1399), ou, comme le dit de Jaucourt, d'attaquer « directement les vices ou les ridicules des hommes [...] qu'elle nomme sans détour, appelant un chat un chat, \& Néron un tyran. [...] La satyre [...] va droit à l'homme. Elle dit: C'est vous, c'est Crispin, un monstre, dont les vices ne sont rachetés par aucune vertu ${ }^{4} \gg$. On peut se demander si Diderot a partagé sans réserve cette conception- là de la satire. En effet le satiriste se caractérise par une certaine cruauté qui cache son plaisir d'être le contempteur des vices par un zèle pour la vertu, remarque finement de Jaucourt, pour qui

l'esprit qui anime ordinairement le satyrique [...] n'est point celui d'un philosophe qui, sans sortir de sa tranquillité, peint les charmes de la vertu et la difformité du vice. Ce n'est point celui d'un orateur qui, échauffé d'un beau zèle, veut réformer les hommes, et les ramener au bien. Ce n'est pas celui d'un poète qui ne songe qu'à se faire admirer en excitant la terreur et la pitié. Ce n'est pas encore celui d'un misanthrope noir, qui hait le genre humain, et qui le hait trop pour vouloir le rendre meilleur. Ce n'est ni un Héraclite qui pleure sur nos maux, ni un Démocrite qui s'en moque : qu'est-ce donc ? Il semble que, dans le coeur du satyrique, il y ait un certain germe de cruauté enveloppé, qui se couvre de l'intérêt de la vertu pour avoir le plaisir de déchirer au moins le vice. ${ }^{5}$

D'après cette typologie, le zèle de Diderot pour la vertu, éclatant dans son théâtre, relèverait de celui du philosophe et de l'orateur. Encore faut-il assumer ce qu'impliquent la défense de la vertu et la condamnation du vice et la volonté de rendre les hommes meilleurs : non seulement l'assurance de savoir ce que signifient les mots de vertu et de vice, la certitude d'être soi-même sinon vertueux du moins d'avoir un goût pour la vertu, 
mais surtout se croire « en état de donner des leçons au public ». Si ce « rôle [est] assez vain » selon Diderot (Ver, IV, 1344), c'est parce que les « leçons » ne peuvent être reçues que par ceux qui en ont le moins besoin et parce que les conduites vertueuses ne proviennent pas de "leçons ", mais d'un ensemble de conditions très difficilement maitrisables par les instituteurs du genre humain. Enfin, en morale les adversaires de Diderot ne sont pas les fanatiques ou les superstitieux, cibles de ses critiques antichrétiennes, mais ceux qui, au nom d'un matérialisme qu'il récuse, justifient le monde tel qu'il va et la force qui le mène. ${ }^{6}$ Cette conception trouve un appui dans l'importance que prend l'enrichissement et le fétichisme de l'or qui devient la valeur de toutes les valeurs : «Et puis toutes nos dissertations sur le vice, la vertu, le vrai, le bon, l'honnête, sont bien frivoles pour ceux qui estiment tout au prix de l'or. » (Ver, V, 472) Enfin, pour être satiriste il faut estimer que « le monde va mal » et s'attribuer « la toute-puissance et la souveraine justice ", c'est-à-dire se croire revêtu de la " charge de lieutenant criminel de l'univers » (Ver, V, 354) explique Diderot aux soeurs Volland. Outre le ridicule qu'il y a à se croire "lieutenant criminel de l'univers ", cette posture ne peut produire que des plaintes infinies et des indignations stériles. On comprend qu'il ne faut pas compter sur lui que pour endosser cette charge et jouer ce rôle.

\section{Les problèmes de la satire}

5 Il en découle que lorsque Diderot écrit un dialogue qu'il qualifie de « satyre » (" satyre seconde »), il ne donne pas une satire comme les autres. Qu'est-ce qui justifie son nom ? La satire est au sens le plus généralement reçu la dénonciation par le ridicule de caractères et de conduites qui s'écartent de valeurs reconnues comme étant bonnes et devant informer les moeurs. ${ }^{7}$ Cela implique plusieurs choses. Concernant les valeurs, la satire suppose que l'on croie qu'elles existent, qu'elles sont généralement reçues, qu'elles relèvent d'un savoir commun et qu'il est possible de s'y rapporter comme à quelque chose de désirable pour faire ressurgir par contraste le mal qui caractérise les vices et par là en dégoûter les hommes en leur faisant honte. Deuxièmement la satire présuppose des lecteurs qui acceptent les implications dont il vient d'être question; et éventuellement des lecteurs prêts à s'amender car sensibles à la honte ou au ridicule. Enfin comme tout genre littéraire, elle repose sur un pacte de lecture : croire dans la sincérité du satiriste, à savoir qu'il croit dans les valeurs, qu'il les connait, qu'il aime la vertu et hait le vice, qu'il a le désir de réformer les individus et les moeurs. Si toutes ces conditions sont remplies, on a affaire à une forme de satire qu'on peut appeler naïve ${ }^{8}$ par opposition à une satire réfléchie et critique. Je souhaite montrer que Le Neveu de Rameau illustre plutôt ce qu'est une satire réfléchie et critique, c'est-à-dire une satire qui s'interrogeant sur ses conditions en vient à les saper et rendre la satire impossible.

6 Pourquoi ce sous-titre alors ? Utilisant les deux sens des mots satire et satyre ${ }^{9}$ on a coutume de souligner à juste titre que ce dialogue emprunte au genre littéraire satirique les traits suivants ${ }^{10}$ : les retournements carnavalesques, le pot-pourri, le mélange des sujets et des niveaux de langue, la présence de l'ordurier, jusqu'à la prose mêlée de musique et de chants qui s'expriment en pantomimes, ${ }^{11}$ et donc la présence du corps et de la voix mis sur le même plan que les discours sérieux. Au-delà encore de ces caractères, la satire seconde repose sur le principe du contraste des contraires (Moi et Lui) qui ne cherchent pas à se résoudre dans un accord supérieur. Ainsi, le décousu de la conversation réfléchit-il la confusion dans la tête du neveu et les troubles ressentis par 
Moi ; la dissemblance qui caractérise Lui est-elle difficilement équilibrée par l'unité de pensée et de ton du philosophe. Aux dissonances du monde introduites dans le dialogue correspondant à la « dissymétrie ${ }^{12}$ » qui définit le neveu, Moi tente d'opposer un style de vie qui retire du plaisir de l'exercice de la connaissance et du spectacle et de la pratique de la morale (84-86). Le seul domaine où le neveu est capable de rencontrer de l'unité et de la louer c'est dans la musique italienne, celle de Duni qui sait « assujettir notre chant à tous les mouvements, à toute les mesures, à tous les intervalles, à toutes les déclamations, sans blesser la prosodie » (131). Le mélange de sentiments ressentis par le philosophe face à son interlocuteur après la grande pantomime des chants (admiration, pitié, ridicule, 127) et le trouble intellectuel et moral qu'il confesse après le récit atroce du Renégat d'Avignon (fuir, rire, s'indigner, 119), résument assez bien ceux de la plupart des lecteurs.

\section{Le satiriste et sa satire}

7 Plus précisément le trouble provient de la constatation que cette oeuvre qui possède la plupart des caractéristiques d'une satire qu'elle fait jouer de façon brillante, en même temps, ne fonctionne pas comme devrait le faire une satire. Il semblerait que ces caractéristiques soient traités de telle sorte qu'on ne puisse pas se prononcer sur le but de la satire et au bout du compte sur son efficacité. De même, on ignore si Diderot avait l'intention de publier ce texte et rien dans sa correspondance ne nous renseigne sur ce qu'il visait en l'écrivant. De ce fait Le Neveu de Rameau est un renfort pour les partisans de l'Intentional fallacy (l'illusion de l'intention). ${ }^{13}$ En tout cas, si le lecteur avait une attente, elle est déçue. Pour le montrer il faut examiner comment le dialogue traite des valeurs (leur existence, leur connaissance, leur désirabilité), du genre de critique (se fait-elle en leur nom ? est-elle de l'ironie, du cynisme ?) et du satiriste (sa sincérité, son engagement de pédagogue et de réformateur). Mais comme c'est dans la figure du satiriste que Diderot a concentré toute la force et les ambiguïtés du dialogue, nous ferons porter sur elle la totalité de nos questions.

On n'a peut-être pas assez insisté sur le fait que cette satire n'est pas menée par l'auteur du livre, ni par le narrateur, Moi, mais par son interlocuteur qui est le véritable satiriste, Lui, alors qu'il compose un caractère digne d'être la cible d'une satire. Et en effet, Moi utilize contre lui des procédés classiques de la satire, consistant à le mettre en contradiction avec lui-même, à dénoncer ses travers en en lui faisant honte, à se moquer de lui. On verra que Diderot a concerté son texte de façon à montrer l'inutilité de ces ressorts.

Par ailleurs, il faut se défaire de l'emprise qu'exercent les analyses trop célèbres du Neveu de Rameau par Hegel dans la Phénoménologie de l'esprit qui font de Lui la conscience de soi déchirée et exprimant la déchirure du monde de la culture dans un langage à la fois spirituel et délirant. ${ }^{14}$ Car on oublie que les références au Neveu de Rameau, faites d'incrustations de morceaux du texte plus que de citations véritables, commencent avant la section sur le rapport aliéné de la conscience de soi à la culture : dans le passage qui décrit la dialectique des rapports des consciences nobles et viles et leur renversement sous le double rapport de la richesse et du pouvoir. ${ }^{15}$ Or ces pages jettent un éclairage sur l'un des problèmes dont traite la satire, le rôle de la richesse dans la disparition des valeurs que le neveu enregistre. On ne voit pas alors que le neveu n'est pas toujours, seulement, une conscience formée par son aliénation dans la culture et que son discours réfléchit l'inversion des valeurs du monde. Il montre qu'il est capable d'argumenter pied 
à pied avec son interlocuteur, il se sert de philosophèmes matérialistes qui sont des citations de Diderot lui-même ${ }^{16}$ il expose une théorie du monde social et a une conscience lucide de son état de parasite et de bouffon des riches, de sa stérilité créatrice et une conscience douloureuse d'être habité par le pire des désirs vains ${ }^{17}$ vouloir être un autre. De même, tous comptes faits, ce n'est pas par ses vices qu'il est intéressant dans la satire : à tout prendre il n'est pas un si grand scélérat qu'il le voudrait en revendiquant trop fort ses vices $(60,67,86,114,119)$ au point de vouloir faire croire qu'il les a tous et à un haut degré de méchanceté. ${ }^{18}$

Plutôt donc que d'insister sur le caractère vicieux du neveu il est préférable de retenir ses qualités. Au fond que pouvons-nous reprocher au neveu sur le plan de la morale ? Il ne paraît pas avoir nui à quiconque, ni s'être fait le complice d'une sale action. Il a beau énumérer complaisamment tous ses défauts, Moi fait remarquer qu'il manque d'unité de caractère et qu'il vacille dans ses principes (115) ce qui dans le vocabulaire de Lui s'appelle être " une espèce[s] de toutes les épithètes la plus redoutable, parce qu'elle marque la médiocrité et le dernier degré du mépris » (133). C'est entendu, ses plus gros défauts sont d'être " fainéant ", de manquer de courage et de préférer " son bonheur à un succès incertain " $(86,142)$, de prendre du plaisir à raconter des horreurs (116-118). Encore ne peut-on pas écarter l'envie d'épater le philosophe. ${ }^{19}$

Alors ce n'est pas seulement le désir de se venger de ses protecteurs qui l'ont chassé qui le pousse à détailler les vices et les ridicules de leur monde et des parasites dont il fait partie. Il est animé par le désir proprement philosophique de dévoiler les mécanismes du monde social qu'il fréquente, de rendre intelligible la nécessité des comportements de ses membres, y compris les siennes. Ou, plus exactement, sa satire bénéficie du fait qu'en se vengeant, en soulignant les ridicules de la " pétaudière » (90), de " la ménagerie » (100) Bertin, en prenant plaisir à déchirer des personnages mauvais (la cohorte des antiphilosophes et des parasites), il fait la théorie de ce qu'il fait et explique le fonctionnement de ce monde. Prenons brièvement un exemple. Lui vient de raconter tout ce que sa situation de factotum de Bertin et Mlle Hus le contraignait à faire chez eux et à l'extérieur pour eux, au prix de ses scrupules, de sa réputation, de sa pudeur, pour recevoir en échange « la table, le lit, l'habit, veste et culotte, les souliers, et la pistole par mois » (108-110). S'engage avec Moi qui réprouve qu'il fasse de « bons contes » sur eux un échange sur la gratitude et l'humiliation. Suit ce vif et bref échange de coups :

Moi : Il est au moins indécent de donner des ridicules à ses bienfaiteurs.

Lui : Mais n'est-ce pas pis encore de s'autoriser de ses bienfaits pour avilir son protégé?

Moi : Mais si le protégé n'étais pas vil par lui-même, rien de donnerait au protecteur cette autorité.

Lui : Mais si les personnages n'étaient pas ridicules par eux-mêmes, on n'en ferait pas de bons contes (110).

Se moquer de son patron est une réaction morale à l'humiliation qu'il subit. Lui interprète correctement le rapport d'échange de services (faire le bouffon - être nourri, vêtu et logé) comme un rapport de domination et d'abaissement contre lequel il n'a que la satire des maitres qui a pour fonction de les rabaisser symboliquement. Du même coup le neveu montre qu'il a compris que dans ce monde règne la force, celle que donne l'argent d'un côté, celle de tromper, calomnier, trahir de l'autre. Et il s'élève à la description de ce qu'il appelle le « pacte tacite » de la société où chacun rend un mal pour le bien reçu (111-112). La conséquence que les habitants de ce monde doivent tirer c'est que si quelqu'un est lésé ce n'est pas parce qu'il y a des méchants mais bien plutôt parce qu'il y a des sots (112). 


\section{Folie et vérité} d'entre deux et pas uniquement le ressentiment du pauvre hère. Être entre-deux c'est avoir été chassé du monde des Bertin et Hus qu'il connait de très près pour en avoir été un élément important : le bouffon et vouloir y retourner sachant que là est le gite, le couvert et la « pistole ». Mais le rôle de bouffon n'est pas compris comme le fait la satire depuis l'Histoire de la folie d'Érasme comme celui qui fait le fou pour dire obliquement la vérité aux uns et aux autres. Le neveu était certes présenté ainsi par le narrateur : « il secoue, il agite ; il fait approuver ou blâmer ; il fait sortir la vérité » (47). Mais s'il se traite lui-même de " fou et de bouffon ", cette fonction est réduite à celle de désennuyer Bertin et sa compagnie $(62,87,108)$. Là n'est donc pas la ressource satirique que l'on pourrait attendre. compris les anecdotes les plus intimes, à laquelle s'ajoute l'exercice d'une sincérité qui expose publiquement ses propres vices et ses pensées. Cette sincérité constitue le cynisme du personnage qui en reconnaissant qu'il cherche à faire son bonheur par les vices qui lui « sont naturels, [...] acquis sans travail, [...] [conservés] sans effort, qui cadrent avec les moeurs de [sa] nation » (87), échappe au ressort de toute satire, la honte. Mais au-delà de l'outrance de ses aveux, il faut souligner que la sincérité est de façon inattendue une vertu de la connaissance et non une vertu au service de la moralité. «J'ignore quel secret je puis avoir pour vous ", répète-t-il (114). « Il n'est pas hypocrite », (136), il est « franc et conséquent » (137), relève Moi. Celui qui dit qu'un chat est un chat et Néron un tyran, ${ }^{20}$ est un satiriste mais si en outre il proclame qu'il est, lui, un composé de vices exhibés sans pudeur, il ne peut que donner des gages d'exactitude : utiliser les mots qui désignent sans gaze c'est apparaître comme vérace. Il dira toujours le vrai des choses (personnes, pratiques, situations, réputations) sans crainte de déplaire. ${ }^{21}$ En revanche, ainsi que l'a finement analysé Bernard Williams, il met à mal l'identité établie par Rousseau entre la sincérité et la bonté : la sincérité n'est plus le signe de la vertu. Elle ne provient pas d'un examen sévère de son intériorité et du désir de véracité pour convaincre autrui de sa bonté. Elle n'implique pas un long travail qui dévoilerait tout d'un coup le sujet.. ${ }^{22} \mathrm{La}$ sincérité n'a pas grand chose à voir avec une histoire de la subjectivité, elle est d'abord au service de son intérêt $(99,104)$. Elle est donc mobilisable comme un mode d'être social obéissant à une stratégie de communication : dire « librement et sans honte ${ }^{23}$ » ce qu'on voit, ce qui est.

Il y a deux modalités de la folie dans Le Neveu de Rameau. Faire le fou, pour distraire des gens qui s'ennuient. Cette folie-là est recherchée et louée et ne comporte pas le risque d'être chassé de la société. Faire le fou en disant une vérité sous une impertinence est dangereux mais reste de l'ordre du mot d'esprit, comme ce fut le cas avec l'abbé de La Porte dans le funeste dîner chez Bertin (105). Mais l'autre folie consiste à dire ce qui est, sur soi et le monde, en exposant la vérité sans apprêt, brutalement, sans tenir compte des usages, des préjugés ou de son intérêt. Être fou, n'est plus "faire le fou ", c'est parler depuis la perte de toute illusion, en particulier morale : « on est dédommagé de la perte de son innocence par celle de ses préjugés " (102). La folie revient à croire que pour dire la vérité il faut être sans innocence et sans préjugé. Alors au-delà de la satire apparaît une forme de pensée scandaleuse qui contient la menace permanente de « cracher le morceau 
", de dire la vérité de tout le monde, ce qui revient à dévoiler ce qui était su de tous et devait resté caché. ${ }^{24}$

\section{Le scepticisme à l'égard des valeurs}

16 La question des valeurs au nom desquelles sont dénoncés les vices se pose alors différemment. Explicitement le neveu est un sceptique à leur égard : elles sont des conventions relatives et variables, leur sens est réversible, être vertueux ou vicieux dépend de l'intérêt à être l'un ou l'autre (104). Mais cette opinion relativiste assez banale à l'époque n'est pas ce qui importe. Le neveu est bien plus radical, « conséquent », quand il récuse l'idée même de valeurs. La litanie des « vanité » qui ponctuent les vertus prônées par Moi (83) sert soit à les démystifier (le patriotisme), soit à montrer qu'elles recouvrent des devoirs que tout le monde cherche à fuir (l'amitié), soit qu'elles cachent ce qui est vraiment indispensable (être riche plutôt que d'avoir un " état »), en somme elles sont contraires à la nature humaine, à la vie sociale. La vérité est dans l'ambivalence où nous sommes face aux vertus : « On loue la vertu, mais on la hait; mais on la fuit; mais elle gèle de froid ; et dans ce monde il faut avoir les pieds au chaud » (87).

C'est pourquoi la question n'est pas tant de connaitre les valeurs et les vertus que de savoir user des vices, à commencer par les siens. Cette relation pragmatique résulte de l'usage des hommes en société et de la lecture les écrits des « moralistes » (102-103), grâce auxquels tout courtisan sait faire " ce qui convient » et, point capital dans ce milieu, éviter le ridicule. Il n'y a pas de valeurs, mais des comportements qui dépendent de l'organisation physiologique et psychologique de chacun, des conventions de langage, de l'habitude. Ce n'est donc pas au nom de ses valeurs qu'il satirise la "ménagerie ». Derrière même la satire qui n'est pas l'élément le plus important, il y a une analyse qui montre la nécessité de leurs agissements. D'où les trois théories du neveu. La société repose sur le pacte tacite (je te fais du bien, tu me trahiras, 111-112) ; les conditions s'entredévorent, d'où la circulation (" restitution ») générale de la richesse $(80)^{25}$; l'universelle pantomime de ceux qui dépendent de quelqu'un pour satisfaire un besoin (148). Elles ne dépendent pas d'une morale ; elles disqualifient les morales prescriptives au nom de normes reposant sur des valeurs, pour présenter d'autres normes qui se rapportent moins à des valeurs qu'à des passions : de la richesse, du pouvoir, de la jouissance des femmes, de la renommée grâce à l'or. Elles sont des descriptions et des explicitations des mécanismes sociaux et des mobiles réels qui engendrent ces passions.

Enfin, dernière théorie découverte par le neveu, la morale commune n'existe pas sans ses exceptions, les « idiotismes moraux » (78-80) qui sont en définitive communs au point d'être la norme sans laquelle la société ne fonctionnerait pas. Dans un raisonnement paradoxal du type de Mandeville, il montre que ces exceptions, ces accrocs à ce que la conscience générale proclame comme étant moral, valent comme une espèce de crédit qui " ne vaut que par l'opinion " (79) qui est nécessaire à la renommée et à l'enrichissement des individus. Il faut se tromper - ou être trompé - sur les talents des individus pour, en les leur faisant crédit, les employer et les payer à faire "bien » ce qu'ils savent faire (80). Là encore il délivre un secret paradoxal, car à la vue de tout le monde : la vie social n'est possible que grâce aux multiples exceptions à la conscience morale générale, mais qu'on ne dit pas ; c'est sur l'opinion que reposent les relations sociales et la création de la renommée nécessite des petits mensonges. 


\section{Le satiriste et sa morale}

19 Le neveu est-il immoral ? À l'égard de la morale commune il est sceptique si on l'interroge, indifférent autrement ou carrément cynique puisqu'il pratique le « mal » en le sachant, en s'en vantant sans remords. En revanche, et contre les apparences, il est le sujet d'une moralité, mieux, il incarne ce qui est pour Diderot la condition naturelle de toute moralité : le sentiment irréfléchi de l'injustice et la révolte qui l'accompagne comme protestation et affirmation du caractère normatif contenu dans ce sentiment. ${ }^{26}$ L'étonnant échange entre Moi et Lui à propos du sentiment de la « dignité » de celui-ci (89-90) fait apparaître une réalité intéressante pour une histoire du sujet moderne, le décrochage de la moralité du sujet, son éthique dira-t-on, de la morale comme code social. Si la moralité s'ancre dans la nature, disons la physiologie de la sensibilité développée en sentiment, elle ne dépend pas de la manière de se conformer à un code normatif extérieur (le code social). Alors que tout l'effort de Diderot consiste à montrer comment la morale sociale exprime la similitude d'organisation, de besoins, de peines, de douleurs, de passions, etc. (voir Ver II, 570, Ver III, 58 et 628-629) ${ }^{27}$ des individus, avec Rameau il construit le cas où cette similitude n'existe pas. Par rapport à la morale commune, Rameau est dans une situation non d'affrontement, de critique ou de transgression : il s'en moque au double sens où il ne s'y intéresse pas et où il la tourne en ridicule. Non pas au nom d'autres valeurs censées être "supérieures ", mais au nom de la bonne coexistence, pour lui, entre ses vices et les moeurs de sa société, bref de son plaisir. Toutefois le sérieux avec lequel il défend son sentiment de dignité, contre toute apparence, montre une conscience de soi que ne laissent pas deviner ses discours et la reconnaissance de son abjection. Pourtant c'est dans le même souffle qu'il rapproche vice et vertu :

Lui : Je veux bien être abject ; mais je veux que ce soit sans contrainte. Je veux bien descendre de ma dignité ...vous riez.

Moi : Oui, votre dignité me fait rire.

Lui : Chacun a la sienne ; je veux bien oublier la mienne, mais à ma discrétion, et non à l'ordre d'autrui (89).

La pensée, le corps et la parole du neveu parcourent selon les situations (moments, lieux, public, intérêts) un espace de possibilités entre deux pôles : d'un côté la parrêsia irréfléchie (" il y a des circonstances où je laisse tout aller sous moi », 107), voire l'aliénation $(126,128)$, de l'autre une conscience de soi qui lui permet de maîtriser ses apparences («Sois hypocrite, si tu veux ; mais ne parle pas comme l'hypocrite. Garde des vices qui te sont utiles; mais n'en aie ni le ton ni les apparences qui te rendraient ridicule ", 103), ${ }^{28}$ de jouer le rôle du flatteur en travaillant « l'attitude admirative du dos » dont il est dérisoirement fier (95). Il y a du comédien paradoxal dans cet effort pour contrôler les apparences qu'en tant que parasite bouffon il doit revêtir pour répondre aux situations où son rôle le place, y compris l'abjection, c'est-à-dire le renoncement à sa dignité. Quand il affirme que s'il est abject c'est qu'il le veut et non parce qu'il est contraint, il dit en définitive qu'il transforme en décision ce qui est nécessité. Tout se joue donc dans sa représentation des choses, dans l'acquiescement donné à ce à quoi il ne peut échapper s'il veut manger. ${ }^{29}$ On a souvent insisté à juste titre sur le cynisme du neveu. ${ }^{30} \mathrm{Il}$ faudrait prêter attention à cette liberté dont il expérimente l'existence en lui et dont il fait l'expérience dans les cas limites où quelque chose qu'on doit appeler sa personnalité est nié. Et il faudrait relever dans cette perspective l'importance que revêt la crainte d'être 
ridicule et, conséquemment, la volonté de l'être à sa guise (« On m'a voulu ridicule, et je me le suis fait ; pour vicieux, nature seul en avait fait les frais ", 104). C'est que prêter à rire signifie qu'on dépend de l'opinion, de la mode et des usages que des envieux et des medíocres retournent contre l'honneur des individus. Le ridicule est la sanction que la singularité reçoit dans les sociétés conformistes et uniformes, explique Duclos dans les Considérations sur les moeurs (1751). ${ }^{31}$ Craindre d'être ridicule, jouer à l'être sont le signe que chez le neveu existe une conscience de soi de sa liberté qui contrebalance son acceptation de l'humiliation. Là est sa tragédie personnelle qu'il a la pudeur, lui impudique (46), de travestir sous le récit burlesque d'une vie de misère sans grand intérêt, mue par les aléas de la faim et de la misère (143-146) et dont il ne se plaint pas. Au philosophe qui défend une Conception morale de la nature, ${ }^{32}$ Lui oppose la vue d'une existence, la sienne, indifférente à la morale.

\section{Le réel vice du neveu}

Quand j'ai suggéré que la " satyre seconde » est une fausse satire, je n'ai pas tenu compte du fait que la prétendue satire du neveu est elle-même, avec le satiriste, l'objet d'une satire première, celle que dresse le narrateur et Moi par ses commentaires, ses apartés. Ce qui est visé par cette satire ne peut être les vices moraux du neveu, on a vu qu'ils sont présentés de façon à échapper à toute satire : on ne peut faire honte à quelqu'un de ce qu'il revendique comme le définissant. La critique glisse sur lui. Ce n'est pas le mauvais goût ou l'absence de goût qui est ridiculisé. On sait que Moi fait l'éloge au contraire de sa sensibilité pour le beau en musique et de la justesse de ses analyses : «comment se fait-il qu'avec un tact aussi fin, une aussi grande sensibilité pour les beautés de l'art musical ; vous soyez aussi aveugle sur les belles choses en morale, aussi insensible aux charmes de la vertu ?» (132; voir aussi 137). Quel alors le genre de vice qui est satirisé par Moi ? Je retiens l'indication donnée par Pascal Engel dans l'article "Penser la satire " selon laquelle à côté des valeurs morales et esthétiques, il faut compter les valeurs intellectuelles, les valeurs qui interviennent dans l'exercice de la pensée théorique. Or il y a quelque chose qui ne va pas chez le neveu, et ce malgré l'effort que je propose pour reconnaître en lui une lucidité cruelle dans la connaissance de sa société et une éthique, en grande partie illusoire, de la liberté de se choisir. Ce qui ne va pas c'est un mépris pour la réflexion $(57,116)$, une affectation d'ignorance $(51,99,125)$, la parodie des notions matérialistes ${ }^{33}$ et un usage seulement pragmatique des grands moralistes Théophraste, La Bruyère, Molière (102). On a tendance à ne pas le relever car le ton d'humour et d'autodérision du personnage fait qu'on ne le prend pas au sérieux. Pourtant Moi souligne les incohérences, les contradictions, les demi-vérités de son interlocuteur, de sorte qu'on comprend qu'il ne le prenne que rarement au sérieux. Mais là encore, Diderot brouille les choses : car en même temps, le neveu proteste contre la manie du philosophe de mieux le comprendre qu'il ne se comprend lui-même («Oh vous voilà, vous autres! Si nous disons quelque chose de bien ; c'est comme des fous, ou des inspirés ; par hasard. Il n'y a que vous autres qui vous vous entendiez. Oui, monsieur le philosophe. Je m'entends ainsi que vous vous entendez ", 54). Ou bien il arrive que le philosophe donne son accord à Lui (en particulier sur les difficultés de la connaissance, 74-75, sur la nécessité d'une bonne éducation, 139). Mais au fond le neveu qui parle beaucoup sur tout, se vante d'ignorer comment il pense : « Moi : Il y a de la raison, à peu près, dans tout ce que vous venez de 
dire. Lui : De la raison! tant mieux. Je veux que le diable m'emporte, si j'y tâche. Cela va, comme je te pousse » (125). Le vice qui est satirisé est le mépris pour la pensée.

\section{Conclusion}

Fausse satire, « la satyre seconde » contient peut-être la vérité suivante. Dans une époque où seule une petite poignée de personnes défendent et aiment les vertus et en en font la condition du bonheur, sans doute parce qu'elles sont constituées ainsi, "gens bizarres " (138) qui imaginent que " le même bonheur est fait pour tous " (82), alors qu'ailleurs on ne s'en préoccupe pas ou bien on est persuadé que la seule valeur qui se respecte est celle de l'or (135), une satire ne sert à rien et la philosophie qui en inspire l'idée, en écrivant Le Neveu de Rameau, doit reconnaître qu'elle est sans force pour modifier la réalité, car quando on est riche on ne peut être ridicule ni déshonoré (84). Si, en outre, le satiriste reconnait qu'il ne se distingue pas de ceux qu'il ridiculise, et que sa seule différence est sa singularité protégée par un sens instinctif de sa valeur, sa satire s'épuise dans la bouffonnerie. La valeur monétaire qui neutralise et assèche toutes les autres, morales, esthétiques, intellectuelles, appelle autre chose que la satire, ce qu'à partir du XIXe siècle on appellera " la critique ». Celle des Lumières est une critique externe, qui applique à ses objets des príncipes et des valeurs qui leur sont étrangers, comme on le voit dans la critique antireligieuse..$^{34}$ Elle fera place à une critique différente en ce que ses valeurs seront immanentes à ses cibles, comme si l'analyse d'un phénomène comprenait en même temps son jugement et, peut-être, la raison de son renversement. Mais il faudra pour cela bien des conditions.

Il est remarquable que les Lumières aient comporté un texte qui n'appartient pas encore pleinement à " la critique ", encore que les trois ou quatre théories du social proposées par le neveu en relèvent, tout en n'étant plus une satire, sinon par ses procédés de dérision, de ridicule, de sacrilège aussi (voir l'élévation du louis d'or parodiant celle de l'hostie, 135), de caricature, de plagiat, de cynisme, bref tout ce qui peut amuser, tout en laissant planer le doute que rien de tout cela n'a de valeur. Quand la critique est devenue inaudible parce que la valeur de l'argent a étendu son emprise sur les modes de pensée et persuadé que la réalité quelle informe est la seule possible, alors il ne reste que des satires et la dénonciation des " fausses valeurs » et d'autorités usurpées au nom de "vraies » qu'on ne cherche même plus à fonder. Reste que toutes n'atteignent pas la qualité du Neveu de Rameau, pensera-t-on, preuve que subsistent la valeur esthétique et de l'exercice du goût.

\section{NOTES}

1. Je laisse de côté la question de savoir s'il est important qu'une oeuvre ait " un sujet », comme le relève Bernard Williams in Vérité et véracité, trad. de Jean Lelaider, Gallimard, 2006, p. 223.

2. Buffon, Histoire naturelle générale et particulière, t. V, [1755], édit. de Jean Piveteau, PUF, 1954, p. 360 . 
3. Sur ce néologisme qui apparaît chez Leibniz (1710), Robinet (1761) et Bonnet (1762), voir Michèle Duchet et Michel Launay, Entretiens sur Le Neveu de Rameau, Nizet, 1967, p. 176-178. Chez Diderot il désigne une originalité très précise, celle qu'induit le fait de dissembler de soi-même comme c'est le cas du neveu (46).

4. Encyclopédie, Article SATYRE de de Jaucourt, XIV, 700.

5. Idem.

6. J'ai insisté sur les adversaires matérialistes de Diderot en morale dans Diderot, la morale et les limites de la philosophie, "L'expérience des vertus ", à paraître.

7. Je suis redevable d'utiles réflexions à l'article de Pascal Engel, «La pensée de la satire », in S. Duval et J.P. Saidah, édit., " Mauvais genre, la satire littéraire ", in Modernités, Presses de l'université de Bordeaux, 2008. Voir également Sophie Duval et Marc Martinez, La satire, A. Colin, 2000, cité par Pascal Engel, que je n'ai pas pu consulter.

8. Je prends le sens de naïf tel qu'il apparaît dans l'opposition entre poésie naïve et poésie sentimentale de Schiller : est dit naïf ce qui est dans un rapport immédiat avec ses conditions tel qu'il ne doute pas de soi-même.

9. Ces deux termes rassemblent les trois caractères qui se retrouvent dans le dialogue : le mélange, le potpourri, la présence de " satyres ", et de l'obscénité et la critique des vices et des ridicules.

10. Voir les présentations du Neveu de Rameau par Michel Delon, op. cit., par Marian Hobson, Genève, Droz, 2013, les préfaces par Jean Varloot, Maurice Roelens et Roland Desné, Éditions sociales, 1972.

11. Ce mélange est rendu par le récit du narrateur qui décrit ce que fait le neveu, et intègre des morceaux chantés ou des sons d'instruments joués par celui-ci. Le mélange est le résultat de l'alternance de paroles et sons qui essaie de rendre leur intrication et la polyphonie de la voix.

12. C'est le terme qu'utilise Michel Delon dans sa présentation de l'oeuvre, op. cit., p. 22.

13. Pour le texte établissant cette thèse, voir Wimsatt et Beardsley, " The Intentional Fallacy ", 1947.

14. Voir Hegel, Phénoménologie de l'esprit, VI. B. I., trad. Bernard Bourgeois, Vrin, 2005, p. 448-450.

15. Ibidem, p. 433-448.

16. J'ai traité de ces questions dans «L'auto-citation dans Le Neveu de Rameau », in Diderot Studies, vol. 34 , à paraître.

17. Je reprends cette notion à Kant en modifiant légèrement son analyse. Les vains désirs, explique Kant, sont ceux par lesquels l'homme est en contradiction avec lui-même : d'un côté il peut par sa simple représentation viser à la production d'un objet ou d'un état, de l'autre avoir conscience que ses forces sont insuffisantes pour produire cet objet ou cet état. La disjonction entre la visée de la chose à réaliser et les moyens mécaniques ou psychologiques de sa réalisation crée de la lassitude mais aussi renouvelle l'effort pour y parvenir - moyen par lequel les hommes mesurent leurs forces de production des choses. Critique de la faculté de juger, Introduction III, note de la 2ème édition, trad. de Ladmiral, de Launay, Vaysse, in Oeuvres philosophiques, II, Gallimard, 1985, p. 931-932.

18. On verra qu'une interprétation en termes de stratégie d'énonciation permet de comprendre ses « aveux » autrement que comme de simples provocations.

19. Il y a là de la part de Diderot un rappel de son propre goût esthétique et de sa curiosité de philosophe pour les grands criminels, qui ne va pas sans interrogation inquiète. Je pense à la question éthique de savoir si la littérature ne se compromet pas et ne compromet pas le lecteur en décrivant des actes dégradants et inhumains, soulevée par John M. Coetzee, dans Elizabeth Costello à propos des tortures infligées par les nazis à des conjurés ayant fomenté un attentat contre Hitler.

20. Voir ci-dessus la citation de Jaucourt, l'article SATYRE, p. 135. 
21. Dans une certaine mesure le neveu fait preuve de la parrêsia à laquelle Michel Foucault a consacré ses derniers cours, Le Gouvernement de soi et des autres et Le Courage de la vérité, GallimardSeuil, 2008 et 2009. Parole libre, effronterie, discours directs, exposition à des disgrâces, sont des traits du parrêsiaste dans la version cynique. Toutefois le fait que le courage de la vérité chez le neveu relève davantage de l'étourderie (le dîner chez Bertin) que d'une vertu constante et le fait que son état de parasite affaiblit sa revendication d'indépendance d'esprit font qu'il est un cynique qui joue le cynique. Notons que M. Foucault signale Le Neveu de Rameau comme « un tournant ", " dans l'histoire de la réflexion sur le cynisme en Occident », dans la mesure où il participe à la genèse de la " fonction de l'art comme cynisme dans la culture », Le Courage de la vérité, ibid., p. 178 et 174.

22. Je suis ici les analyses très suggestives de Bernard Williams, Vérité et véracité, op. cit., p. 226-227.

23. Ibidem, p. 226.

24. Avoir dit la vérité de tout le monde est sans doute ce qui a constitué, paradoxalement, le scandale de De l'esprit d'Helvétius. J'ai analysé cette question dans " Helvétius, l'idée d'une science de l'homme et la politique », in Matérialistes français du XVIIIe siècle, La Mettrie, Helvétius, d'Holbach, sous la direction de Sophie Audidière, Jean-Claude Bourdin, Jean-Marie Lardic, Francine Markovits, Yves Charles Zarka, PUF, 2006, p. 167-192.

25. En parlant de « restituer» $(80,81)$ pour parler de la circulation de la richesse par flatteries, aumônes et dépenses somptuaires qui supposent toutes des riches et des gueux liés par des échanges inégaux, le neveu utilise un terme savant pour désigner une pratique improductive et parasitaire : forme cynique de la satire.

26. Sur cette question je signale «La notion de nature dans Le Neveu de Rameau (la faim et la philosophie morale de la nature) », in Dix-huitième siècle, n 45, « La nature », éd. par Colas Duflo, 2013.

27. On se reportera également à l'édition des Fragments politiques échappés du portefeuille d'un philosophe, édition et notes de Gianluigi Goggi, Hermann, 2011, p. 111-112.

28. Cette maxime est reprise comme règle d'éducation pour son fils : « le point difficile [...] ce n'est pas de donner à son enfant des vices qui l'enrichissent, les ridicules qui le rendent précieux aux grands ; [...] c'est de lui marquer la juste mesure, l'art d'esquiver à la honte, au déshonneur, aux lois. » (137)

29. Certains pourront penser que c'est là une preuve de sa mauvaise foi, tentative pitoyable de sauver la face devant le philosophe qui lui en impose quand même. Je ne les suivrais pas sur ce terrain, faisant l'hypothèse charitable au neveu de la réalité de son drame : vouloir être un autre, un musicien créateur célébré et opulent grâce à son art et incapable de l'être, pour des raisons qu'il examine lucidement.

30. Voir de Jacques D’Hondt, « Le Cynisme de Rameau », in RDE, 36, 2004.

31. Voir Charles Pinot Duclos, Considérations sur les moeurs, 1751, chapitre VIII, p. 207-209.

32. Je me permets de renvoyer une fois de plus à « La notion de nature dans Le Neveu de Rameau (la faim et la philosophie morale de la nature)», op. cit.

33. Voir ci-dessus note 17.

34. Voir l'analyse saisissante qu'en donne Hegel dans la Phénoménologie de l'esprit, VI, II, « Le combat des Lumières contre la superstition », op. cit. p. 465 et suiv. 


\section{RÉSUMÉS}

La morale occupe une place centrale dans l'oeuvre de Diderot et dans Le Neveu de Rameau. La morale a un double sens: les moeurs, les opinions et les comportements valorisés dans une société et la science des moeurs. À côté des études savantes ou apologétiques (religieuses), la satire offre un moyen pour comprendre les normes et les valeurs morales, en dénonçant leur transgression par des vicieux. Le Neveu de Rameau est une « satyre ». L'article analyse le genre de satire qu'est ce dialogue. Il apparaît que si d'un côté c'est Lui, le vicieux qui est la cible de la satire, de l'autre c'est lui qui fait la satire d'un milieu social qui nie les valeurs de la morale commune. L'hypothèse est que ce dispositif vise à montrer les limites de la philosophie en ce qui concerne la morale.

\section{INDEX}

Keywords : Morals is the main concern of Diderot's works and in Le Neveu de Rameau. Morals means two things: customs, opinions and behaviors that a society values, and the science of the customs. There is many ways to lead the knowledge of Morals, but the satire is a good way to understand and defend the moral values by denouncing those who break the morality, the perverts. Le Neveu de Rameau is a " satyre ». This article focuses on the kind of satire this dialogue is. It seems that if on one side the pervert (Lui) is the target of the satire, on the other one il is him who offers a vigorous satire of a society which denies the values of common morality. The hypothesis is that this double satire aims to show the limits of philosophy as regards the morality.

Mots-clés : morale, satire, philosophie, limites de la philosophie

\section{AUTEUR}

\section{JEAN-CLAUDE BOURDIN}

Université de Poitiers, France. jclbourdin@gmail.com

Professeur émérite de l'Université de Poitiers. Ses travaux portent sur trois domaines: les Lumières, Diderot et le matérialisme; Hegel, Marx, Arendt, Foucault, Althusser; la politique (la guerre, la réconciliation, religion et politique, la critique). A publié sur ces questions de nombreux articles, des ouvrages et dirigé des ouvrages collectifs. En dernière date: Althusser, une lecture de Marx, PUF, 2008, Les Politiques de réconciliation, L'Harmattan, 2013, « Machiavel philosophe sans guillemets » in La Pensée, n. 382 , « Althusser 25 ans après », avril-juin 2015.

Professor emeritus of the University of Poitiers (France). His investigation concern three domains: The Enlightenment, Diderot, the materialism; Hegel, Marx, Arendt, Foucault, Althusser; and several issues in politics (war, violence, reconciliation, religion and politics, the critical stand). Last publications: Althusser, une lecture de Marx, PUF, 2008, Les Politiques de réconciliation, L'Harmattan, 2013, « Machiavel philosophe sans guillemets » in La Pensée, n. ${ }^{\circ} 382$, « Althusser 25 ans après », avril-juin 2015. 